three periods: $0.78(0.75-0.80), 0.77(0.76-0.79)$, and 0.75 (0.72-0.78), respectively $(P=0.334)$. Overall, 6,373 (19.9\%) patients had DM. At a median (interquartile range) follow-up of $5.0(3.5-5.0)$ years, $543(8.5 \%)$ and 1270 (5.0\%) patients with and without DM developed HCC. The AUROC $(95 \%$ $\mathrm{CI})$ of PAGE-B score to predict HCC at 5 years was lower in DM patients $(0.68[0.66-0.70])$ than in non-DM patients (0.79 [0.78-0.80]) $(P<0.001) .651(10.2 \%)$ DM patients were classified as low risk; their 5-year HCC cumulative incidence (95\% CI) was $2.1 \%(1.1 \%-3.6 \%)$, which is higher than the threshold of cost-effective HCC surveillance suggested by international clinical guideline, i.e. $0.2 \%$ annually. 6,536 (25.6\%) non-DM patients were classified as low risk; their 5year HCC cumulative incidence $(95 \% \mathrm{CI})$ was $0.5 \%(0.4 \%$ $0.7 \%$ ) (figure 1).

Conclusions PAGE-B score is accurate across the years to predict HCC, yet has a lower performance in DM patients. HCC risk persists and HCC surveillance is still cost-effective in DM patients classified as low risk by PAGE-B score.

\section{IDDF2020-ABS-0206 PREDICTORS OF RESPONSE TO THERAPY WITH TERLIPRESSIN AND ALBUMIN IN PATIENTS WITH CIRRHOSIS AND HEPATORENAL SYNDROME - ACUTE KIDNEY INJURY (HRS-AKI) ACCORDING TO NEW INTERNATIONAL CLUB OF ASCITES (ICA) CRITERIA}

Jatin Agrawal ${ }^{*}$, Ashish Kumar, Anil Arora, Praveen Sharma, Vikas Singla, Naresh Bansal, Shrihari Anikhindi. Institute of Liver, Gastroenterology and Pancreaticobiliary Sciences, Sir Ganga Ram Hospital, India

\subsection{6/gutjn--2020-IDDF.16}

Background HRS-AKI or HRS -1 carries high short-term mortality in patients with advanced cirrhosis. Recently ICA has proposed new definition criteria for HRS, and at present, we lack literature on the response rate and predictors of response of terlipressin according to this new definition. So, we aimed to evaluate the response rate of terlipressin and factors affecting the response rate according to this new HRS-AKI definition

Methods We performed a prospective study on 114 cirrhotic patients with HRS- AKI diagnosed according to ICA definition 2015 from August 2018 to April 2020 using terlipressin and albumin. Baseline clinical and biochemical details were noted. Response was defined as improvement in serum creatinine within $0.3 \mathrm{mg} / \mathrm{dl}$ of baseline (if baseline s.cr $<1.5 \mathrm{mg} / \mathrm{dl}$ ) or $<1.5 \mathrm{mg} / \mathrm{dl}$ (if baseline s.cr $>1.5 \mathrm{mg} / \mathrm{dl}$ ). Further responder and non-responder were followed up to 90 days or death. Univariate and multivariate logistic regression was applied to detect predictors of response. Survival analysis was used to determine 90 days of survival.

Results Among 114 HRS-AKI patients, the median age was 52.5 years, and $83.3 \%$ were male. Response to terlipressin was seen in $70(61.4 \%)$ patients. On subgroup analysis, the response rate in acute decompensation (AD) was seen in 37 $(78.7 \%)$ patients and in acute on chronic failure (ACLF) was seen in $32(47.7 \%)$ patients. Independent predictive factors of response to therapy were serum creatinine before start of terlipressin therapy (odds ratio, 0.390; 95\% confidence interval, $0.195-0.780 ; \mathrm{P}=0.008)$ and baseline child-pugh score (CTP) (odds ratio, 0.584; 95\% confidence interval, 0.382-0.894; $\mathrm{P}$ $=0.013)$. Response to therapy was associated with improved
Kaplan-Meier Survival Estimates According to Response

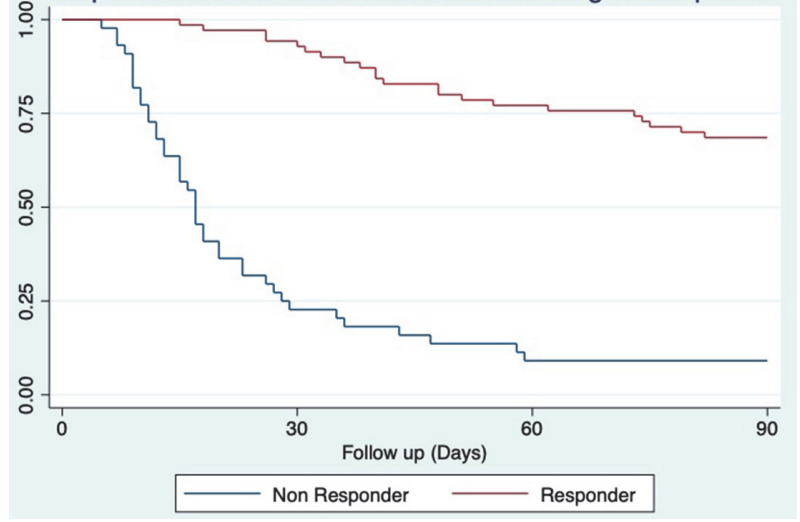

Abstract IDDF2020-ABS-0206 Figure 190 days survival among responder and non- responder groups

90 days of survival compared to patients with non-response (69.56\% vs $10 \%, \mathrm{P}<0.00001)$.

Conclusions Serum creatinine before the start of terlipressin and CTP predicts response to terlipressin in HRS-AKI. The response rate was better with the new definition of HRS. The subset of the population with higher grades of ACLF showed poor response. Therefore early transplantation should be considered in patients of HRS with a low likelihood of response (figure 1).

\section{Basic gastroenterology}

\begin{tabular}{ll}
\hline IDDF2020-ABS-0034 & NETWORK PHARMACOLOGY ANALYSIS TO \\
& UNCOVER THE POTENTIAL MECHANISMS \\
& OF LYCIUM BARBARUM ON COLORECTAL \\
& CANCER
\end{tabular}

Yi Lu*, Jiachen Sun, Minhui Hu, Xianhe Kong, Weijie Zhong. Department of Gastrointestinal Endoscopy, the Sixth Affiliated Hospital, Sun Yat-sen University, China

\subsection{6/gutjnl-2020-IDDF.17}

Background Studies have shown that extracts from lycium barbarum could play a protective role against colorectal cancer (CRC) cells. We used the network pharmacology approach to establish the effects of lycium barbarum on CRC and to predict core targets and their biological functions, pathways, and mechanisms of action.

Methods We obtained the active compounds and their targets in lycium barbarum through Traditional Chinese Medicine System Pharmacology Database (TCMSP), retracted the CRC targets from Malacards, TTD, GeneCards, and DisGeNET, and chosen the overlapped targets as the candidate targets. After protein-protein interaction (PPI) network analysis, 20 with the highest node degree were selected as the core targets, and their enrichment and pathway were analyzed. Furthermore, iGEMDOCK was employed to validate the compound-target association.

Results Eventually, 103 overlapped targets were chosen as the candidate targets. Targets with the top 20 highest node degree were selected as the core targets. Gene Ontology (GO) enrichment analysis indicated that the core targets were significantly enriched in regulation of cell proliferation, extracellular space, cytokine receptor binding, and so on. Kyoto Encyclopedia of 